\title{
Conservação dos recursos pesqueiros da Costa Atlântica Brasileira através da ótica do conhecimento ecológico local: uma revisão sistemática (2011 - 2021)
}

\author{
Conservation of the fishing resources of the Brazilian Atlantic Coast through the perspective of
}

local ecological knowledge: a systematic review (2011 - 2021)

Conservación de los recursos pesqueros de la Costa Atlántica Brasileña desde la perspectiva del

conocimiento ecológico local: una revisión sistemática (2011 - 2021)

Recebido: 01/08/2021 | Revisado: 04/08/2021 | Aceito: 07/08/2021 | Publicado: 13/08/2021

\author{
Leidiane Priscilla de Paiva Batista \\ ORCID: https://orcid.org/0000-0003-3027-6393 \\ Universidade Federal do Ceará, Brasil \\ E-mail: leidianepriscilla|@gmail.com \\ Fábio de Oliveira Matos \\ ORCID: https://orcid.org/0000-0001-9275-9361 \\ Universidade Federal do Ceará, Brasil \\ E-mail: fabiomatos@ufc.br
}

\begin{abstract}
Resumo
Os pescadores artesanais são reconhecidamente portadores de valiosos conhecimentos sobre os recursos que manejam. Portanto, é importante que as vivências e saberes desenvolvidos por estas populações sejam consideradas na gestão dos ambientes por elas manejados. O objetivo deste estudo foi compreender como os pescadores artesanais influenciam a conservação dos recursos pesqueiros na costa atlântica brasileira. Para isso, revisou-se artigos que abordaram populações pesqueiras de regiões litorâneas brasileiras e a conservação desses recursos. Essa revisão demonstrou a necessidade de estudos que busquem compreender essa relação, em especial nas regiões Sul e Norte do país e que abranjam a pesca de outros grupos taxonômicos além de peixes ósseos. Em sua maioria, os trabalhos, alertaram para o potencial dos conhecimentos ecológicos locais em contribuir com a conservação dos recursos manejados. Muitos dos saberes detidos pelos pescadores são desconhecidos pela comunidade científica e precisam ser investigados e incluídos na gestão da zona costeira brasileira.
\end{abstract}

Palavras-chave: Pescadores artesanais; Etnoconservação; Etnoecologia; Ambientes litorâneos; Gestão costeira.

\begin{abstract}
Artisanal fishermen are recognized as carriers of valuable knowledge about the resources they manage. Therefore, it is important that the experiences and knowledge developed by these populations be considered in the management of the environments they manage. The objective of this study was to understand how artisanal fishermen influence the conservation of fishery resources on the Brazilian Atlantic coast. For this, we reviewed articles that addressed fishing populations of Brazilian coastal regions and the conservation of these resources. This review demonstrated the need for studies that seek to understand this relationship, especially in the southern and northern regions of the country and that cover fisheries of other taxonomic groups besides bony fish. Most of the studies pointed out the potential of local ecological knowledge to contribute to the conservation of managed resources. Much of the knowledge held by fishermen is unknown by the scientific community and needs to be investigated and included in the management of the Brazilian coastal zone.
\end{abstract}

Keywords: Artisanal fishermen; Ethnoconservation; Ethnoecology; Coastal environments; Coastal management.

\section{Resumen}

Los pescadores artesanales son reconocidos como portadores de valiosos conocimientos sobre los recursos que gestionan. Por lo tanto, es importante que las experiencias y los conocimientos desarrollados por estas poblaciones se tengan en cuenta en la gestión de los entornos que gestionan. El objetivo de este estudio era comprender cómo influyen los pescadores artesanales en la conservación de los recursos pesqueros de la costa atlántica brasileña. Para ello, se revisaron los artículos que abordaban las poblaciones pesqueras de las regiones costeras brasileñas y la conservación de estos recursos. Esta revisión demostró la necesidad de realizar estudios que busquen entender esta relación, especialmente en las regiones Sur y Norte del país y que abarquen las pesquerías de otros grupos taxonómicos además de los peces óseos. La mayoría de los estudios han señalado el potencial de los conocimientos ecológicos locales para contribuir a la conservación de los recursos gestionados. Gran parte de los conocimientos de 
los pescadores son desconocidos por la comunidad científica y deben ser investigados e incluidos en la gestión de la zona costera brasileña.

Palabras clave: Pescadores artesanales; Etnoconservación; Etnoecología; Ambientes costeros; Gestión costera.

\section{Introdução}

É marcante na história da humanidade um progressivo processo de ruptura do homem com o seu entorno (Santos, 2005), tornando-se hegemônico o pensamento de que a sociedade não faz parte da natureza. De tal modo que, o modelo conservacionista estadunidense, almejante da natureza selvagem (intocada por grupos humanos), foi importada para os países do Sul (Diegues, 2000). Com isso, desenvolveu-se um modelo conservacionista excludente das questões sociais e culturais, em que os conhecimentos ecológicos e práticas socioeconômicas das populações locais eram desconsiderados (Diegues, 2014) e essas populações não eram ouvidas e chamadas a participar no processo de gestão dos seus próprios territórios.

Em um movimento oposto, a etnoconservação busca a inserção das comunidades tradicionais e locais na gestão de ecossistemas e recursos naturais, partindo da noção de que a dimensão humana é natureza. Deste modo, é incabível dissociar conservação ambiental e sociedade.

Absorto a este contexto, os estudos para a conservação dos recursos pesqueiros são comumente fundamentados na avaliação biológica dos estoques (Braga et al., 2018), sendo mister considerar as vivências e saberes gestados pelas populações que vivem desses recursos e incluí-los na gestão participativa dos mesmos. Os conhecimentos adquiridos por meio de observações e manejo dos recursos naturais ao longo de suas vidas podem fornecer informações preciosas sobre as populações e ecossistemas marinhos e serem utilizadas para orientar práticas eficientes de gestão, monitoramento e manejo.

A maior parte da produção pesqueira brasileira decorre da pesca artesanal, que é formada por pequenas aldeias pontuais e dispersas, a maioria das quais segue regras locais quanto ao uso do ambiente natural e do espaço aquático (Begossi, 2010). Estes pescadores são reconhecidamente portadores de valiosos conhecimentos sobre os recursos manejados.

O objetivo deste estudo foi compreender como os pescadores artesanais influenciam a conservação dos recursos pesqueiros na costa atlântica brasileira. Para isso, revisou-se artigos que abordaram populações pesqueiras de regiões litorâneas e a conservação desses recursos. Buscou-se ainda caracterizar como esses trabalhos estão distribuídos ao longo do tempo e regiões do país, assim como quais são os ambientes marinhos e os principais grupos taxonômicos explorados pelas populações estudadas.

\section{Metodologia}

Realizou-se revisão sistemática sobre a relação dos pescadores brasileiros com a conservação dos recursos pesqueiros na zona costeira entre os anos de 2011 e 2020. Para análise e seleção da literatura científica, foi utilizado o protocolo PRISMA (Preferred Reporting Items for Systematic Reviews and MetaAnalyses) (Moher et al., 2011), que consiste de quatro etapas (Figura 1) que auxiliam metodologicamente revisões sistemáticas e metanálise.

Os dados foram obtidos a partir de busca nas bases de dados Scielo, Science Direct e Scopus. Em todas as plataformas, foi utilizado o mecanismo de "busca avançada", restritas entre período de janeiro de 2011 a junho de 2021 . As palavras-chaves utilizadas foram "conservação", "pescadores" e "Brasil", localizadas no título, resumo ou palavras-chave. Utilizou-se o operador booleano "e" para que todos os termos fossem incluídos na pesquisa. Realizou-se busca de artigos nas línguas portuguesa e inglesa. 
Figura 1. Fluxograma referente ao procedimento de seleção de referências bibliográficas (Protocolo Prisma).

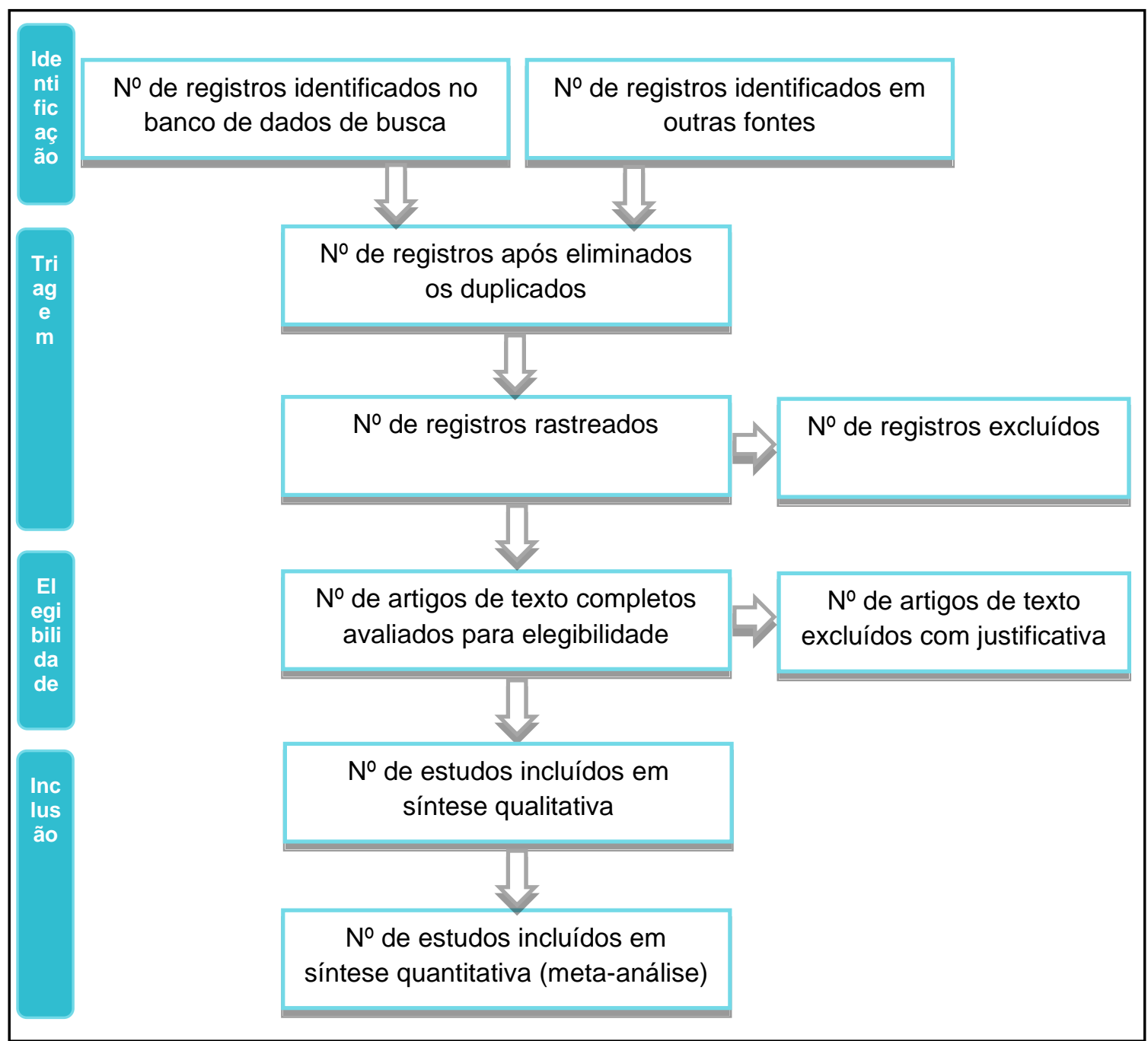

Fonte: Autores.

De acordo com os critérios de elegibilidade previamente estabelecidos (Quadro 1), os artigos foram analisados e, quando cumpridos os requisitos, incluídos na pesquisa. Após leitura dos artigos, as informações relevantes foram coletadas por meio de planilha eletrônica, registrando-se para cada artigo: autor, ano de publicação, revista, título, localidade de estudo, objetivos, metodologia, resultados e observações gerais.

Quadro 1. Critérios de elegibilidade para a seleção das referências bibliográficas relacionadas ao protocolo PRISMA.

\section{Critérios de Inclusão}

Artigos realizados com pescadores marinhos ou estuarinos

Artigos com pesquisa de cunho etnobiológico

Artigos com abordagem dos recursos pesqueiros

Artigos relevantes para o tema

\section{Critérios de Exclusão}

Livros, relatórios, tese, dissertações, monografias e resumos

Artigos que não discutiram conservação dos recursos naturais

Fonte: Autores.

Para análise de dados quantitativa, considerou-se as variáveis: ano de publicação; região e estado das localidades; ambiente de pesca (marinho ou estuarino); e grupo taxonômico dos recursos pesqueiros capturados pelas populações de 
pescadores estudadas (peixes ósseos; elasmobrânquios e crustáceos). Ainda, realizou-se análise qualitativa para compreensão de como os pescadores artesanais influenciam na conservação dos recursos pesqueiros na zona costeira brasileira. Como alguns manuscritos abrangeram, ocasionalmente, mais de uma categoria dessas variáveis, o número total de registros por vezes supera o número total de artigos analisados.

\section{Resultados e Discussão}

Considerando-se o período de tempo selecionado para a revisão, a análise identificou 165 registros, distribuídos da seguinte forma nas bases de dados pesquisadas: Scopus - 95; Science Direct - 59; Scielo: 11. Após supressão das duplicatas e aplicação dos critérios de elegibilidade, selecionou-se dezenove artigos que abordaram a relação de pescadores artesanais de ambientes costeiros com a conservação de recursos pesqueiros no Brasil.

Conforme Figura 2, não houveram registros de publicação em alguns anos. A maioria das publicações ocorreram entre 2012 e 2015. Esses dados nos apontam sobre a necessidade de que mais estudos de cunho etnobiológico e etnoecológico busquem discutir como as populações investigadas influenciam e/ou contribuem para a conservação dos recursos pesqueiros, indicando caminhos a serem traçados na gestão e manejo desses recursos.

Figura 2. Números de artigos de cunho etnobiológico que abordaram a relação entre pescadores artesanais de ambientes costeiros e a conservação de recursos pesqueiros no Brasil entre 2011e 2021.

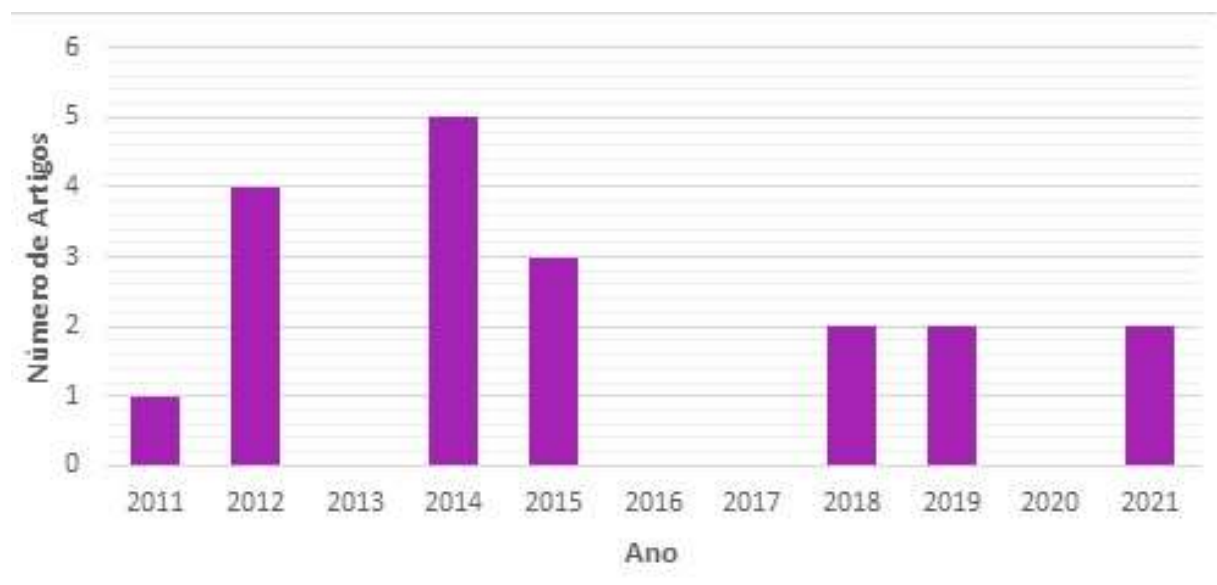

Fonte: Autores.

Ao longo da zona costeira brasileira, os estudos foram realizados principalmente com pescadores de áreas marinhas (80\%). Uma pequena parcela abrangeu pescadores de ambientes estuarinos (20\%). Foram encontrados registros de publicação em onze dos dezessete estados brasileiros com área litorânea (Figura 3). Em termos regionais, o maior número de artigos convergiu na região Nordeste (15), seguida da região Sudeste (9). Esperava-se que os registros convergissem nestas regiões, visto que elas possuem núcleos de estudo e pesquisa em etnociências que são referências nacionais para a área. Na região Sul, houve apenas um registro e na região Norte, onde os estudos concentraram-se em populações de pescadores continentais, nenhum registro foi encontrado, revelando a demanda em compreender a etnoconservação em áreas costeiras nestas regiões do país. 
Figura 3. Distribuição por estado brasileiro de artigos publicados de cunho etnobiológico que abordaram a relação entre pescadores artesanais de ambientes costeiros e a conservação de recursos pesqueiros no Brasil entre 2011 e 2021 . Como alguns estudos abrangeram mais de um estado, o número total de registros supera o número total de artigos analisados.

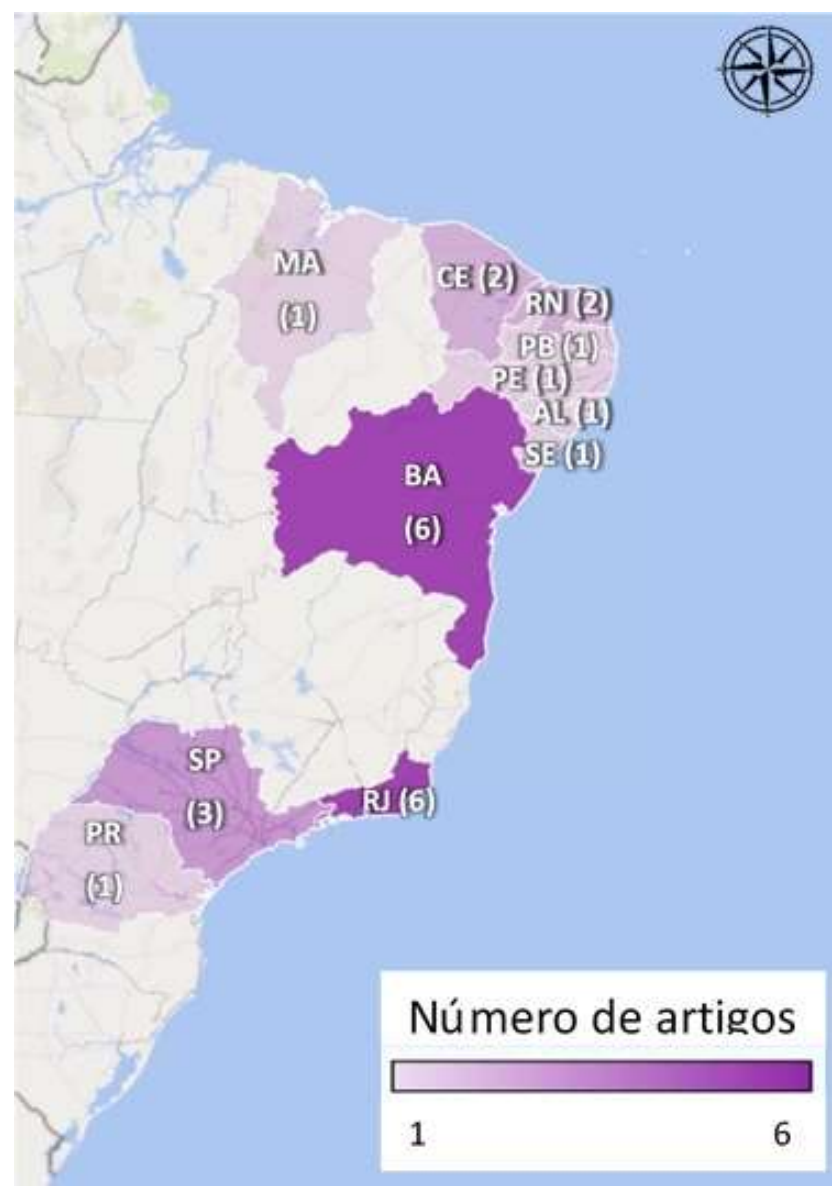

Fonte: Autores.

A respeito do grupo taxonômico dos recursos pesqueiros explorados (Quadro 2), a maioria dos trabalhos (16) abrangeu a pesca de peixes ósseos. Três estudos pesquisaram pescadores de Elasmobrânquios, enquanto outros três artigos incluíram em suas análises pescadores de Crustáceos, mais especificamente caranguejo, camarão e lagosta. O trabalho que incluiu pescadores de lagosta na coleta de informações, por escolha metodológica, não diferenciou esse grupo na análise de dados (cf. Silva; Lopes, 2015). 
Quadro 2. Grupos taxonômicos dos recursos pesqueiros e localização dos estudos selecionados através da metodologia Prisma.

\begin{tabular}{|c|c|c|c|c|}
\hline Registro & Localização & $\begin{array}{l}\text { Peixes } \\
\text { Ósseos }\end{array}$ & $\begin{array}{l}\text { Elasmo- } \\
\text { brânquios }\end{array}$ & Crustáceos \\
\hline Barbosa Filho et al., 2014 & $\mathrm{BA}$ & & $\checkmark$ & \\
\hline Begossi et al., 2011 & $\mathrm{AL}, \mathrm{BA}, \mathrm{RJ}$ e SP & $\checkmark$ & & \\
\hline Begossi et al., 2012 & $\mathrm{RJ}$ & $\checkmark$ & & \\
\hline Bezerra et al., 2012 & PB & $\checkmark$ & & \\
\hline Braga et al., 2018 & RJ & $\checkmark$ & & \\
\hline Cantanhede et al., 2018 & MA & $\checkmark$ & & \\
\hline Cortes; Zappes; Beneditto, 2014 & $\mathrm{RJ}$ & & & $\checkmark$ \\
\hline Erler; Lima; Schiavetti, 2015 & BA & $\checkmark$ & & \\
\hline Ferreira et al., 2014 & BA & $\checkmark$ & & \\
\hline Giareta et, 2021 & PR & & $\checkmark$ & \\
\hline Martins; Medeiros; Hanazaki, 2014 & BA & $\checkmark$ & & \\
\hline $\begin{array}{l}\text { Morado; Andrade-Tubino; Araújo, } \\
2021\end{array}$ & $\mathrm{RJ}$ & $\checkmark$ & & \\
\hline Messias et al., 2019 & SE & $\checkmark$ & & \\
\hline Pinto; Mourão; Alves, 2015 & PE e CE & $\checkmark$ & $\checkmark$ & \\
\hline Ramires; Rotundo; Begossi, 2012 & SP & $\checkmark$ & & \\
\hline Silva, Oliveira; Schiavetti, 2014 & $\mathrm{RN}$ & $\checkmark$ & & \\
\hline Silva; Lopes, 2015 & CE e RN & $\checkmark$ & & $\checkmark$ \\
\hline Silvano, Begossi, 2012 & SP & $\checkmark$ & & \\
\hline
\end{tabular}

Fonte: Autores.

Diante do exposto, observou-se a demanda por estudos que englobem a relação entre pescadores e a conservação de recursos pesqueiros poucos estudados para o litoral brasileiro, como elasmobrânquios e crustáceos. O enforque em profissionais que usam apetrechos seletivos de pesca, permitem a compreensão da interação dos pescadores com as espécies capturadas e do estado de conservação dos recursos explotados, assim explicitam as estratégias de intervenção necessárias para a garantia dos estoques.

Quanto a seletividade na pesca, observou-se que as técnicas seletivas capturam os indivíduos na idade adulta ou no tamanho permitido por lei, enquanto as técnicas generalistas capturam os espécimes em diversos tamanhos e fases de desenvolvimento (Bezerra et al., 2012). É importante ainda destacar a tendência de pescadores mais antigos e que realizam pesca seletiva cumprirem mais as regras de manejo em comparação com pescadores mais jovens e que não usam artes de pesca seletivas, demonstrando que a preocupação com a conservação é maior nos profissionais que realizam a atividade a mais tempo e que a dependência de um recurso específico torna o pescador mais vulnerável ao colapso do mesmo (Silva; Lopes, 2015).

Na pesca da Sardinella brasiliensis, os pescadores do Sul do Brasil apresentaram comportamentos conservacionistas moderados, sendo que os armadores demonstraram maior conhecimento ecológico da espécie do que os pescadores que não possuem barcos, dado que se justifica pela maior responsabilidade dos primeiros nas práticas pesqueiras (Braga et al., 2018). Quanto aos conhecimentos sobre Epinephelus itajara, uma espécie que apesar de ter a pesca proibida pela legislação brasileira e ser de rara captura, registrou-se no Nordeste do país que ela está marcadamente presente no imaginário popular, podendo ser usada como espécie-bandeira em projetos de conservação da biodiversidade marinha (Ferreira et al., 2014).

No Sudeste, os pescadores observaram indícios de que uma população de Mugil liza está se reproduzindo em uma estação do ano diferente da época reprodutiva comumente registrada para essa espécie, assinalando a necessidade de confirmação científica, podendo, deste modo, contribuir com políticas de conservação e manejo dessa espécie (Morado; Andrade-Tubino; Araújo, 2021). Achado semelhante foi encontrado para os Lutjanídeos na costa leste nordestina (Messias et al., 2019). 
No concernente a conservação de elasmobrânquios, percebeu-se que o desconhecimento da biologia reprodutiva das diferentes espécies de tubarões por parte dos pescadores, leva a atitudes que prejudicam a renovação das populações, como captura de fêmeas prenhas ou abaixo da idade reprodutiva (Barbosa-Filho et al., 2014). Logo, são necessários a elaboração e aplicação de projetos de educação ambiental para sensibilização e conscientização dos pescadores sobre a importância da conservação desses peixes. A exemplo do que foi observado no sul do país, quando a inclusão de pescadores em atividades de pesquisa levou ao aumento do comprometimento dos membros da comunidade com a soltura compensatória de elasmobrânquios de baixo valor comercial ou que não atingiram o tamanho mínimo de captura, além de que muitos pescadores passaram a desembarcar os animais capturados inteiros (sem decapitar ou eviscerar), contribuindo para a identificação das espécies e monitoramento do pescado (Giareta et al., 2021).

Catadores de caranguejo do Sudeste brasileiro realizam práticas de manejo tradicional que promovem a etnoconservação da espécie $U$. cordatus, como reconhecimento das diferenças sexuais, que permite poupar as fêmeas; habilidade de estimar o tamanho dos caranguejos, proporcionando a seleção dos indivíduos maiores; e a rotação das áreas de manguezais exploradas, executando o pousio (Côrtes; Zappes; Beneditto, 2014).

Ampliando os estudos sobre as possibilidades de contribuição das populações pesqueiras para a conservação de crustáceos, constatou-se no Nordeste do país, que os pescadores de camarão associaram a diminuição na abundância desse pescado com o aumento do esforço de pesca e com o ruído e poluição causados por barcos (Martins; Medeiros; Hanazaki, 2014). Estas percepções podem subsidiar a gestão desse recurso, uma vez que eles identificaram potenciais causas para a queda na produtividade desses animais.

A respeito dos tabus alimentares em grupos locais que sobrevivem da pesca, seu estudo pode apontar indícios sobre o uso dos recursos, pois aliviam a pressão pesqueira em algumas espécies, enquanto exercem maior pressão sobre outras (Ramires; Rotundo; Begossi, 2012). Entretanto, é necessário que pesquisas futuras investiguem de modo mais aprofundando como esse tipo de restrição alimentar influencia na conservação dos recursos.

No tocante a participação na gestão das áreas manejadas, observou-se a necessidade de meios que atraiam a adesão dos pescadores, como a inclusão dos conhecimentos locais nos planos de manejo (Erler; Lima; Schiavetti, 2015; Pinto; Mourão; Alves, 2015; Silva; Lopes, 2015; Martins; Medeiros; Hanazaki, 2014; Silva; Oliveira; Schiavetti, 2014; Silvano; Begossi, 2012). Outra estratégia seria o Pagamento por Serviços Ambientais - como ocorre no Seguro Defeso - para incentivar a cogestão dessas áreas e para promover a conservação da biodiversidade, visto que a maioria dos pescadores são pobres e enfrentam, por vezes, o dilema das áreas marinhas onde atuam serem impactadas por arrastões realizados pela pesca industrial, levando-os a considerar a conservação como um fardo que lhes é imposto (Begossi et al., 2012; Begossi et al., 2011).

Além da inclusão na gestão, outra medida apresentada é a conscientização das comunidades sobre a importância de zelar pelos recursos pesqueiros (Cantanhêde et al., 2018), pois a funcionalidade da medida de restringir a captura do pescado para que os recursos não se esgotem não é tão clara para as populações que sobrevivem da pesca. A exemplo de trabalho realizado por Bueno; Schiavetti (2019) no Nordeste, onde os professionais entrevistados não compreenderam o valor da Unidade de Conservação para os estoques de peixes. Isto indica a importância do diálogo entre os gestores das áreas protegidas e as comunidades pesqueiras envolvidas, buscando compreender quais são as demandas e dificuldades enfrentadas por elas e desenvolver medidas mitigadoras para as mesmas.

De forma geral, os trabalhos apontaram o potencial dos conhecimentos ecológicos locais em contribuir para a conservação dos recursos manejados, uma vez que os pescadores são portadores de valiosos saberes sobre a biologia e ecologia das espécies capturadas, por vezes desconhecidos ou pouco aprofundados pela academia. A etnociência dessas populações são subsídios para a tomada de decisões sobre a gestão dos recursos pesqueiros, devendo-se considerar a gestão participativa das áreas ocupadas e exploradas pelas mesmas. 


\section{Conclusão}

Essa revisão sistemática demonstrou a necessidade de que mais estudos busquem compreender a relação de pescadores artesanais de ambientes costeiros com a conservação de recursos pesqueiros no Brasil, em especial nas regiões Sul e Norte. É preciso também que concentrem esforço na pesca de outros grupos taxonômicos além de peixes ósseos. A inclusão, nas pesquisas, de profissionais que usam apetrechos seletivos de pesca contribui para a compreensão da interação dos pescadores com as espécies capturadas e do estado de conservação dos recursos explotados.

O conjunto de informações construídos nas práticas cotidianas e na reflexão sobre as vivências permitem aos pescadores assumir atitudes que promovam a conservação dos recursos manejados, como o reconhecimento das diferenças sexuais ou a percepção de que a fêmea de uma espécie está grávida, que permitem a eles decidir se a devolve ao mar em caso de captura; ou saber o tamanho em que determinada espécie alcança a maturidade sexual, podendo escolher armadilhas de pesca que capturem indivíduos maiores.

Os conhecimentos dos pescadores artesanais têm o potencial de contribuir com a gestão de áreas costeiras e marinhas, em especial de áreas protegidas. Urge a inclusão desses saberes e experiências nos planos de manejo, assim como é preciso favorecer estratégias que atraiam os pescadores a participar em um modelo de cogestão, por meio do qual eles sintam que seus conhecimentos são valorizados e possam participar das tomadas de decisão. Sugere-se a aplicação de projetos de educação ambiental, que dialoguem com essas comunidades e possam ajudá-los a compreender a importância da conservação e como ela pode contribuir para a proteção dos estoques pesqueiros.

Os trabalhos que abordaram a etnoconservação, em sua maioria, alertaram para o potencial dos conhecimentos ecológicos locais em contribuir com a conservação dos recursos manejados. Muitos dos saberes detidos pelos pescadores são desconhecidos pela comunidade científica e precisam ser investigados e incluídos na gestão da zona costeira brasileira. Deste modo, sugere-se que trabalhos etnoecológicos e etnobiológicos futuros enfoquem em como as populações pesqueiras influenciam na conservação dos recursos manejados, englobando regiões poucos estudadas, conforme apresentado nesse estudo. Destacamos a importância de estudar sobre a etnoconservação e a pesca de outros grupos taxonômicos além dos peixes ósseos, como crustáceos, moluscos e peixes cartilaginosos.

\section{Referências}

Barbosa-Filho, M. L. V. et al. (2014). "Shark is the man!": ethnoknowledge of Brazil's South Bahia fishermen regarding shark behaviors. Journal of Ethnobiology and Ethnomedicine, 10 (1), 1-14.

Begossi, A. (2010). Small-scale fisheries in Latin America: management models and challenges. Mast, 9 (2), 7-31.

Begossi, A. et al. (2012). The Paraty artisanal fishery (Southeastern Brazilian coast): ethnoecology and management of a social-ecological system (SES). Journal of Ethnobiology and Ethnomedicine, 8 (22), 1-18.

Begossi, A. et al. (2001). Ethnobiology of snappers (Lutjanidae): target species and suggestions for management. Journal of Ethnobiology and Ethnomedicine, 7 (11), 1-22.

Bezerra, D. M. M. et al. (2012). Influence of tides and winds on fishing techniques and strategies in the Mamanguape River Estuary, Paraíba State, NE Brazil. Anais da Academia Brasileira de Ciências, 84 (3), 775-787.

Braga, H. O. et al. (2018). Conserving brazilian sardine: fisher's attitudes and knowledge in the Marine Extractive Reserve of Arraial do Cabo, Rio de Janeiro State, Brazil. Fisheries Research, 204 (8), 402-411.

Bueno, P. F. \& Schiavetti, A. (2019). The influence of fisherman scale in the resilience of socio-ecological systems: an analysis using Q methodology. Ocean and Coastal Management, 169 (3), 214-224.

Cantanhêde, L. G. et al. (2018). Environmental perception of fishermen: use and conservation of fisheries resources. Biota Neotropica, 18 (4), e20170510.

Côrtes, L. H. O.; Zappes, C. A. \& Beneditto, A. P. M. (2014). Ethnoecology, gathering techniques and traditional management of the crab Ucides cordatus Linnaeus, 1763 in a mangrove forest in South-eastern Brazil. Ocean and Coastal Management, 93 (7), 129-138.

Diegues, A. C. (2014). The role of ethnoscience in the build-up of ethnoconservation as a new approach to nature conservation in the tropics. Revue d'ethnoécologie, 6 (2), 1-15. 
Research, Society and Development, v. 10, n. 10, e322101018952, 2021 (CC BY 4.0) | ISSN 2525-3409 | DOI: http://dx.doi.org/10.33448/rsd-v10i10.18952

Diegues, A. C. (2000). Etnoconservação da Natureza: enfoques alternativos. In: A. C. Diegues (Org.). Etnoconservação: novos rumos para a proteção da natureza nos trópicos (pp. 1-46). Hucitec: São Paulo.

Erler, D. M.; Lima, D. P. \& Schiavetti, A. (2015). Ecological fishing networks in a marine protected area: one possibility for evaluating objectives. Ocean and Coastal Management, 104 (2), 106-114.

Ferreira, H. M. et al. (2014). Local ecological knowledge of the artisanal fishers on Epinephelus itajara (Lichtenstein, 1822) (Teleostei: Epinephelidae) on Ilhéus coast - Bahia State, Brazil. Journal of Ethnobiology and Ethnomedicine, 10 (1), 1-15.

Giareta, E. P. et al. (2021). Fishermen's participation in research and conservation of coastal elasmobranchs. Ocean and Coastal Management, 199 (1), 105421 .

Martins, I. M.; Medeiros, R. P. \& Hanazaki, N. (2014). From fish to ecosystems: the perceptions of fishermen neighboring a southern brazilian marine protected area. Ocean and Coastal Management, 91 (5), 50-57.

Messias, M. A. et al. (2019). Ethnoecology of Lutjanidae (snappers) in communities of artisanal fisheries in northeast Brazil. Ocean and Coastal Management, $181(15), 104866,2019$

Moher, D. et al. (2011). PRISMA statement. Epidemiology, 22 (1), 128-133.

Morado, C. N.; Andrade-Tubino, M. F. \& Araújo, F. G. (2021). Local ecological knowledge indicates: There is another breeding period in the summer for the mullet Mugil liza in a Brazilian tropical bay. Ocean and Coastal Management, 205 (7), 105569, 2021.

Pinto, M. F.; Mourão, J. S. \& Alves, R. R. N. (2015). Use of ichthyofauna by artisanal fishermen at two protected areas along the coast of Northeast Brazil. Journal of Ethnobiology and Ethnomedicine, 11 (1), 1-32.

Ramires, M.; Rotundo, M. M. \& Begossi, A. (2012). The use of fish in Ilhabela (São Paulo/Brazil): preferences, food taboos and medicinal indications this research was conducted in three communities of artisanal. Biota Neotropica, 12 (1), 21-29.

Santos, M. (2005). A questão do meio ambiente: desafios para a construção. Geotextos, 1 (1), 139-152.

Silva, E. F.; Oliveira, J. E. L. \& Schiavetti, A. (2014). Local Ecological Knowledge (LEK) of artisanal fishing in the state sustainable development Reserve Ponta do Tubarão - RN, Brazil. Boletim do Instituto de Pesca, 40 (3), 355-375.

Silva, M. R. O. \& Lopes, P. F. M. (2015). Each fisherman is different: Taking the environmental perception of small-scale fishermen into account to manage marine protected areas. Marine Policy, 51 (1), 347-355.

Silvano, R. A. M. \& Begossi, A. (2012). Fishermen's local ecological knowledge on southeastern Brazilian coastal fishes: Contributions to research, conservation, and management. Neotropical Ichthyology, 10 (1), 133-147 